Article

\title{
Research on the Optimal Configuration of Regional Integrated Energy System Based on Production Simulation
}

\author{
Tao Shi ${ }^{1, *(\mathbb{O})}$, Ruan-Ming Huang ${ }^{2}$ and Cang-Bi Ding ${ }^{1}$ \\ 1 Institute of Advanced Technology, Nanjing University of Posts and Telecommunications, \\ Nanjing 210023, China; dcb19960926@163.com \\ 2 Economic and Technological Research Institute, State Grid Shanghai Municipal Electric Power Company, \\ Shanghai 200122, China; hrms@163.com \\ * Correspondence: shitao@njupt.edu.cn
}

Received: 23 June 2020; Accepted: 23 July 2020; Published: 25 July 2020

\begin{abstract}
This paper is focused mainly on the production simulation method of a regional integrated energy system under random scenarios for optimal configuration. First, the cooling, heating, and electric load demand of the regional integrated energy system is described quantitatively in the form of time series, as well as the power characteristics of renewable energy, such as wind power and photovoltaic power generation. Then, a typical scenario set of regional integrated energy system configurations considering the random probability characteristics is established through scene clustering. Second, considering the power output characteristics and cost factors of different types of distributed energy, the corresponding technical and economic quantitative model is established. Third, a multi-objective production simulation model of a regional integrated energy system considering configuration constraints and operation constraints is proposed with economic and environmental protection as the main objectives. Finally, the accuracy and effectiveness of the above methods are verified based on a case study of actual engineering.
\end{abstract}

Keywords: integrated energy system; optimal configuration; scene clustering; production simulation

\section{Introduction}

In recent years, with economic and social sustainable development and renewable energy sources such as wind power and solar energy utilization technology of large-scale popularization and application, energy production and consumption patterns have undergone major transformation. The traditional single-form energy subsystem has been gradually aggregated into a new integrated energy system (IES) through physical coupling and information interconnection. The new generation of IES is a network physical system composed of various primary and secondary energy production, transmission, consumption, and energy storage devices with electric power as its core and the power grid as the main carrier [1]. Multi-energy complementary and coordinated optimization are the typical characteristics of IES [2]. According to the energy supply scale and geographical distribution, IES can be classified into a user-side small integrated energy system, a regional integrated energy system (RIES), and a cross-regional integrated energy system [3]. Among them, the optimization of RIES for industrial parks has become the focus of current research.

In Reference [4], the mathematical model of the optimization of the combined cooling, heating, and power (CCHP) system is discussed, and an algorithm of the system design based on the linear programming model is proposed to improve the convergence rate. In reference [5], a mixed-integer linear programming model was used to design an IES for households, and the types of main equipment and installed capacity were determined. In references [6,7], the genetic algorithm solves the optimal 
design problem of distributed IES. In reference [8], considering the thermal performance and economic performance of the equipment, the impact of the carbon tax on the installed capacity of the CCHP system equipment is revealed. In reference [9], the impact of system environmental indicators on the whole life cycle planning of the system is considered. In Reference [10], a fuzzy theoretical model of the multi-objective design of a CCHP system was proposed. In reference [11], a two-layer frame structure suitable for the optimal design of a distributed energy system in China is proposed, which comprises integrated energy equipment type selection and equipment capacity selection based on load changes. In reference [12], a framework for the optimal planning and operation of IES is proposed, and the objectives and system constraints of integrated energy planning are pointed out. In reference [13], the CCHP system construction cost model is built considering system reliability, economic benefits, and environmental benefits. At present, the research on the optimal configuration of the integrated energy system mainly focuses on the small-scale CCHP system, and it focuses on the integrated design of the cooling, heating, and electrical equipment of a single system. There is also few research studies on the optimal configuration of an RIES for a large industrial park, and the traditional deterministic power planning method is usually used.

Moreover, due to the random fluctuations in the power of intermittent distributed energy in RIES, the configuration decision of distributed energy type and capacity in RIES planning and construction is difficult. For this reason, this paper proposes a method for the optimal configuration of RIES based on a clustering of time-series scenarios and production simulation. First, this method uses time-series cluster analysis to quantitatively describe the time series and random characteristics of the configuration scenario of RIES and establishes the corresponding configuration scenario set. Second, considering the constraints of configuration and operation, a production simulation model of RIES is established with the objectives of economy and environmental protection. Finally, based on the configuration scenario set of an RIES, the expected value of the associated configuration scheme is obtained through production simulation. In this paper, the accuracy and effectiveness of the above methods are verified based on the analysis of actual engineering cases.

\section{Configuration Scenario Quantitative Description}

The configuration scenarios of the RIES include an energy supply scenario and an energy demand scenario. Among them, due to the influence of natural resource conditions and user demand, the output and load power of renewable energy generation represented by wind power generation (WG) and photovoltaic power generation (PV) have a certain randomness, which are collectively referred to as "random sources" here.

In this paper, time-series vectors are used to describe the power characteristics of random sources within a certain time period.

Suppose there are $m$ random sources in the system, and the active power (outflow is positive, inflow is negative) of the $k$-th random source is $P_{i j}$ at the $i$-th time period. Then, in the whole time cycle $T$, the system "random source" time-series scenario can be expressed as:

$$
S=\left[\begin{array}{ccccc}
P_{11} & \cdots & P_{1 k} & \cdots & P_{1 K} \\
\vdots & \ddots & \vdots & \ddots & \vdots \\
P_{i 1} & \cdots & P_{i k} & \cdots & P_{i K} \\
\vdots & \ddots & \vdots & \ddots & \vdots \\
P_{N 1} & \cdots & P_{N k} & \cdots & P_{N K}
\end{array}\right], i=1,2, \cdots N, k=1,2, \cdots K
$$

where $S$ is a matrix of $K$ "random source" time-series scenarios; $T$ is the total simulation duration; $N$ is the total number of time steps; $\Delta t=T / N$ is time-step duration.

Suppose the sample number of the random source time-series scenario is $K$; then, the configuration scenario set of the RIES is $\left\{S_{k}\right\}, k=1,2, \cdots, K$. Considering the similarity and difference between the samples of each time-series scenario, the clustering algorithm (C4.5, K-means, support vector machines 
algorithm, etc.) was used to obtain the typical configuration scenarios of the $k^{\prime}$ RIES, where $K^{\prime}<K$. For the typical scenario of the $d$-th type, the sample mean of the scenario of the same type is used as the quantitative description of the scenario of the type, namely:

$$
\begin{gathered}
S_{d}^{\prime}=\frac{\sum_{k \in d} S_{k}}{k_{d}}, d=1,2, \cdots, K^{\prime} \\
q_{d}=k_{d} / K, d=1,2, \cdots, K^{\prime}
\end{gathered}
$$

where $S_{d}^{\prime}$ is the matrix of the $d$-th typical scenario; $k_{d}$ is the number of samples included in the $d$-th typical scenario; and $q_{d}$ is the corresponding distribution probability of the $d$-th typical scenario in the sample space.

\section{Distributed Energy Technology Model}

\subsection{Renewable Energy}

The renewable energy in an RIES includes mainly WG and PV. For WG, the power output characteristics of the wind turbine can be expressed by a piecewise linear function [14]:

$$
P_{W G}=\left\{\begin{array}{cl}
0 & 0 \leq v \leq v_{c i} \\
\frac{v-v_{c i}}{v_{r}-v_{c i}} \cdot P_{r} & v_{c i}<v<v_{r} \\
P_{r} & v_{r}<v<v_{o c} \\
0 & v>v_{o c}
\end{array}\right.
$$

where $P_{W G}$ is the output power of the wind turbine; $P_{r}$ is the rated power of the wind turbine; $v_{c i}$ is the cut-in wind speed of the wind turbine; $v_{r}$ is the rated wind speed; $v_{o c}$ is the cut-out wind speed; and $v$ is the wind speed at the hub height of the wind turbine.

For PV, the power output characteristics are related to the ambient temperature and irradiance, and the engineering approximate calculation formula can be used as follows [14]:

$$
P_{p v}=\frac{P_{S T C} G_{A C}\left[1+k\left(T_{c}-T_{f}\right)\right]}{G_{S T C}}
$$

where $P_{p v}$ is the output power of PV; $G_{A C}$ is the irradiation intensity; $P_{S T C}$ is the maximum output power under standard test conditions (irradiation intensity $1 \mathrm{~kW} / \mathrm{m}^{2}$, ambient temperature $25^{\circ} \mathrm{C}$ ); $G_{S T C}$ is the light intensity under standard test conditions, and its value is $1 \mathrm{~kW} / \mathrm{m}^{2} ; k$ is the power temperature coefficient, and its value is $-0.47 \% / \mathrm{K} ; T_{\mathcal{c}}$ is the operating temperature of the photovoltaic module; and $T_{r}$ is the reference temperature, with a value of $25^{\circ} \mathrm{C}$ (which needs to be converted to kelvins for calculation).

\subsection{Fuel-Based Distributed Energy}

Fuel-based distributed energy includes mainly diesel generators, gas turbines, etc. Its energy consumption and power output characteristics are as follows [14]:

$$
V_{M T}=\sum_{i=1}^{N} \frac{P_{e, i} \cdot \Delta t}{\eta_{M T} \cdot L}
$$

where $V_{M T}$ is the fuel consumption; $P_{e, i}$ is the actual generating power at the $i$-th time period; $\eta_{M T}$ is the power generation efficiency; and $L$ is the low heating value per unit volume of fuel.

For combined heat and power (CHP) units, the relationship between power generation and heating capacity is quantified by the thermoelectric ratio, as shown below [14]:

$$
\eta_{p q, i}=\frac{P_{e, i}}{Q_{h, i}}
$$

where $\eta_{p q, i}$ is the thermoelectric ratio; $P_{e, i}$ is the actual generating power; $Q_{h, i}$ is the actual heating power; and subscript " $i$ " means time-step index. 
On the basis of $\mathrm{CHP}$, the absorption chiller is used to recycle the waste heat of the cogeneration unit of heat and power to make refrigeration, forming a CCHP system, as shown in Figure 1, where BESS means battery energy storage system.

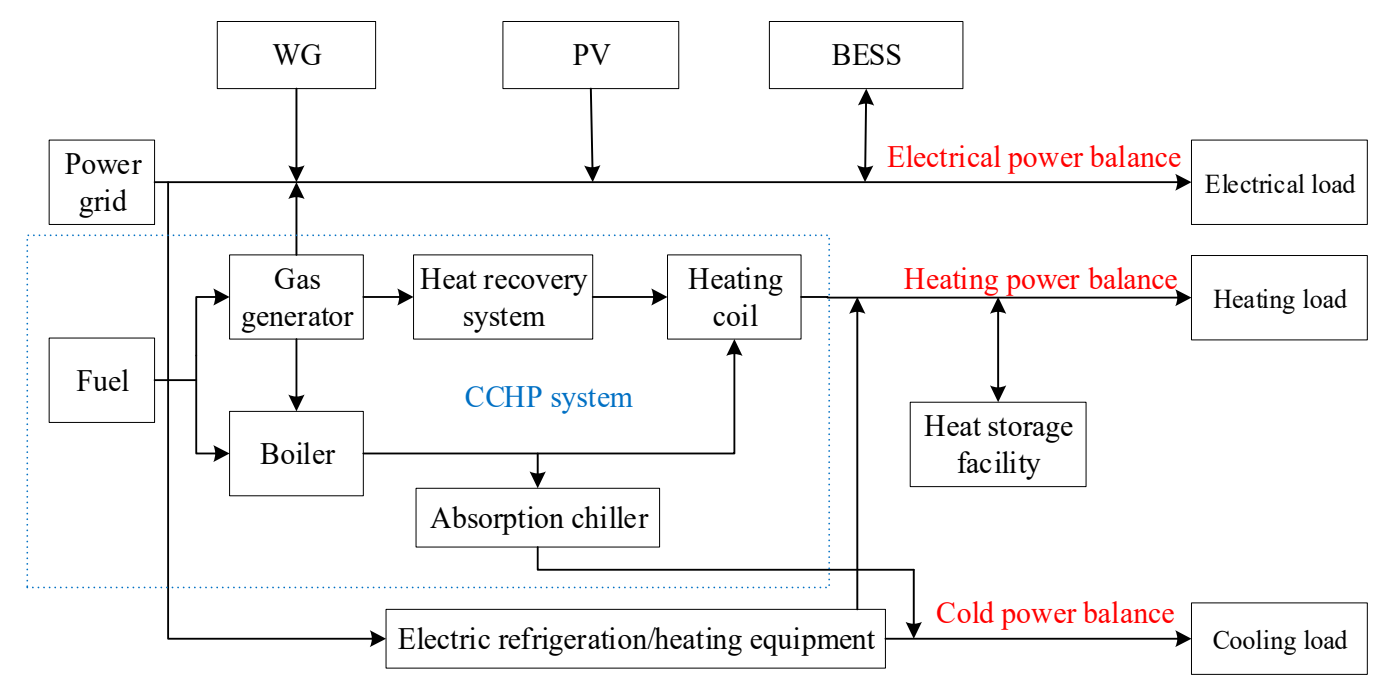

Figure 1. Schematic diagram of the cooling, heating, and power (CCHP) system.

The output characteristics of the power generation, heating capacity, and cooling capacity of the CCHP system are as follows [14]:

$$
\left\{\begin{array}{l}
Q_{M T, i}=P_{e, i} \cdot \frac{1-\eta_{M T}-\eta_{0}}{\eta_{M T}} \\
Q_{h, i}=Q_{M T, i} \cdot K_{h} \\
Q_{c, i}=Q_{M T, i} \cdot K_{c}
\end{array}\right.
$$

where $P_{e, i}$ is the generating power at the $i$-th time period; $\eta_{M T}$ is the power generation efficiency; $Q_{M T, i}$, $Q_{h, i}$, and $Q_{c, i}$ are the residual heat, heating capacity, and cooling capacity of the exhaust gas at the $i$-th time period respectively; and $\eta_{0}, K_{h}$, and $K_{c}$ are the heat dissipation coefficient, heating coefficient, and cooling coefficient, respectively.

\subsection{Energy Storage Facilities}

The energy storage facilities in an RIES mainly include BESS and heat storage facilities. The energy storage and release characteristics are as follows [14]:

$$
\begin{gathered}
\text { Energy storage : } \operatorname{Soc}_{i}=(1-\delta) \cdot \operatorname{Soc}_{i-1}+\frac{P_{c} \cdot \Delta t \cdot \eta_{c}}{E_{c}} \\
\text { Energy release }: \operatorname{Soc}_{i}=(1-\delta) \cdot \operatorname{Soc}_{i-1}-\frac{P_{d} \Delta t}{E_{c} \eta_{d}}
\end{gathered}
$$

where $S o c_{i}$ is the remaining energy of the energy storage system at the end of the $i$-th time period; $S o c_{i-1}$ is the remaining energy of the energy storage system at the end of the (i-1)-th time period; $\delta$ is the self-discharge/heat dissipation rate of the energy storage system; $P_{c}$ and $P_{d}$ are the power of the energy storage and release of the energy storage system, respectively; $\eta_{c}$ and $\eta_{d}$ are the energy storage and release efficiency of the energy storage system, respectively; and $E_{\mathcal{c}}$ is the rated capacity of the energy storage system.

\section{Distributed Energy Economy Model}

The economic factors of distributed energy mainly include the construction fixed investment capital, fuel cost, and operation and maintenance cost. When environmental benefits are taken into 
account, the corresponding carbon emission cost should also be taken into account. The carbon emission cost depends mainly on the carbon emissions of each distributed energy source and the current carbon trading price. For different types of distributed energy, the quantitative description of its economic factors is also different:

(1) For renewable energy, such as wind power and photovoltaics, the fuel cost is zero, the carbon emissions are zero, and the predominant costs are the fixed investment cost and operation and maintenance cost.

(2) For conventional units such as gas and coal, the fuel cost and carbon emission cost are as follows:

$$
\begin{gathered}
g_{i j}\left(P_{i j}\right)=P_{i j} \cdot \Delta t \cdot C_{j} \\
h_{i j}\left(P_{i j}\right)=P_{i j} \cdot \Delta t \cdot V_{a r, j} \cdot C_{a r}
\end{gathered}
$$

where $g_{i j}$ is the fuel cost; $h_{i j}$ is the carbon emission cost; $P_{i j}$ is the power generation power; $C_{j}$ is the production cost per unit energy; $C_{a r}$ is the carbon emission trading price; and $V_{a r, j}$ is the carbon emission per unit energy. Subscript " $i$ " means time-step index; subscript " $j$ " means the $j$-th unit.

(3) For the external public power grid connected to the RIES, the power supply cost and carbon emission cost are as follows:

$$
\begin{array}{r}
g_{i}\left(P_{l i}\right)= \begin{cases}P_{l i} \cdot \Delta t \cdot C_{i n, i} & P_{i} \geq 0 \\
P_{l i} \cdot \Delta t \cdot C_{o u, i} & P_{i}<0\end{cases} \\
h_{i}\left(P_{l i}\right)= \begin{cases}P_{l i} \cdot \Delta t \cdot \eta_{0} \cdot C_{a r} & P_{l i} \geq 0 \\
0 & P_{l i}<0\end{cases}
\end{array}
$$

where $P_{l i}$ is the exchange power of the tie line between the RIES and the external public grid (input is positive, output is negative); $C_{i n, i}$ is the electricity selling price of the external public grid; $C_{o u, i}$ is the on-grid electricity price of the RIES; subscript " $i$ " means time-step index; $C_{a r}$ is the carbon emission trading price; $\eta_{0}$ is the carbon emission corresponding to the unit electricity quantity of the public power grid; and the carbon emission amount caused by the standard coal combustion corresponding to the $\mathrm{kWh}$ is calculated here. This paper takes $\eta_{0}=0.997$; thus, the corresponding carbon dioxide emission of $1 \mathrm{kWh}$ of electricity is $0.997 \mathrm{~kg}$.

\section{Production Simulation Model Algorithm}

\subsection{Objective Function}

Based on the energy management and scheduling strategy of the RIES, a multi-objective optimization model for RIES production simulation was established with consideration of the production and operation costs, equipment depreciation costs, and carbon emissions of the RIES, as shown in the following formula:

$$
\min f=\lambda_{1} \cdot\left(C_{g}+C_{m}\right)+\lambda_{2} \cdot C_{h}
$$

where $C_{g}$ is the production and operation cost; $C_{m}$ is the depreciation cost of the equipment; $C_{h}$ is the cost of carbon emissions; $\lambda_{1}$ is the weight coefficient of the economic target; and $\lambda_{2}$ is the weight coefficient of the environmental protection target.

Here,

$$
\begin{gathered}
C_{g}=\sum_{i=1}^{N} \sum_{j=1}^{M} g_{i j}\left(P_{i j}\right) \\
C_{h}=\sum_{i=1}^{N} \sum_{j=1}^{M} h_{i j}\left(P_{i j}\right) \\
C_{m}=\sum_{i=1}^{N} \sum_{j=1}^{M} \frac{C_{I N L, j} \cdot C_{R F}(l, r)}{8760}
\end{gathered}
$$




$$
C_{R F}(l, r)=\frac{r(1+r)^{l}}{(1+r)^{l}-1}
$$

where $M$ is the number of various distributed energies participating in optimization; $P_{i j}$ is the active power output; $g_{i j}$ is the energy production cost; $h_{i j}$ is the carbon emission cost; $C_{I N I, j}$ is the initial investment cost; $C_{R F}(l, r)$ is the capital recovery factor; $r$ is the annual average depreciation rate; and $l$ is the engineering design period. Subscript " $i$ " means time-step index; subscript " $j$ " means the $j$-th distributed energy.

\subsection{Operating Constraints}

During the RIES operation, it is necessary to meet constraints such as power balance, thermal balance, upper and lower limits of unit output, and upper and lower limits of energy storage capacity.

(1) Electrical power balance constraint

$$
P_{L, i}+P_{l o s s, i}+P_{x, i}=\sum_{n e=1}^{N E} P_{n e, i}+\sum_{n c h=1}^{N C H} P_{n c h, i}+P_{s, i}
$$

where $P_{L, i}$ is the electrical load demand in the $i$-th time period; $P_{\text {loss }, i}$ is the electric power loss in the $i$-th time period; $P_{x, i}$ is the interactive power with the external power grid in the $i$-th time period (input is positive, output is negative); $P_{n e, i}$ is the generating power of the ne-th power source in the $i$-th period; $P_{n c h, i}$ is the generating power of the $n c h$-th CCHP unit in the $i$-th time period; $P_{s, i}$ is the charge/discharge power of the electric energy storage facility in the $i$-th period (discharge is positive, charge is negative); $N E$ is the number of power sources; and NCH is the number of CCHP units.

(2) Heating power balance constraint

$$
Q_{h, i}+Q_{l o s s, i}=\sum_{n h=1}^{N H} Q_{n h, i}+\sum_{n c h=1}^{N C H} Q_{n c h, h, i}+Q_{h s, i}
$$

where $Q_{h, i}$ is the heating load demand; $Q_{\text {loss }, i}$ is the heating power loss; $Q_{h s, i}$ is the heat absorption/ emission power of the heat storage facility (heat emission is positive, heat absorption is negative); $Q_{n h, i}$ is the heating power of the $n h$-th power source; $Q_{n c h, h, i}$ is the heating power of the $n c h$-th CCHP unit; and $\mathrm{NH}$ is the number of pure heating units. Subscript " $i$ " means time-step index.

(3) Cold power balance constraint

$$
Q_{c, i}=\sum_{n c=1}^{N C} Q_{n c, i}+\sum_{n c h=1}^{N C H} Q_{n c h, c, i}
$$

where $Q_{c, i}$ is the heating load demand; $Q_{n c, i}$ is the cooling power of the $n c$-th electric refrigeration equipment; $Q_{n c h, c, i}$ is the cooling power of the $n c h$-th CCHP unit; NC is the number of pure refrigeration units. Subscript " $i$ " means the $i$-th time period.

(4) Power constraint of pure power supply equipment

$$
P_{n e, \min } \leq P_{n e, i} \leq P_{n e, \max }
$$

where $P_{n e, \max }$ and $P_{n e, \min }$ are the upper and lower limits of the ne-th electricity source, respectively.

(5) Power constraint for pure heating equipment

$$
Q_{n h, \min } \leq Q_{n h, i} \leq Q_{n h, \max }
$$

where $Q_{n h, \max }$ and $Q_{n h, \min }$ are the upper and lower limits of the output of the $n h$-th heating equipment, respectively.

(6) Power constraints of pure refrigeration equipment

$$
Q_{n c, \min } \leq Q_{n c, i} \leq Q_{n c, \max }
$$

where $Q_{n c, \max }$ and $Q_{n c, \min }$ are the upper and lower limits of the output of the $n c$-th refrigeration equipment, respectively. 
(7) Coupling operation constraint of CCHP

$$
\begin{aligned}
& P_{n c h, \min }\left(Q_{n c h, h}, Q_{n c h, c}\right) \leq P_{n c h, i} \leq P_{n c h, \max }\left(Q_{n c h, h}, Q_{n c h, c}\right) \\
& Q_{n c h, h, \min }\left(P_{n c h}, Q_{n c h, c}\right) \leq Q_{n c h, h, i} \leq Q_{n c h, h, \max }\left(P_{n c h}, Q_{n c h, c}\right) \\
& Q_{n c h, c, \min }\left(P_{n c h}, Q_{n c h, h}\right) \leq Q_{n c h, c, i} \leq Q_{n c h, c, \max }\left(P_{n c h}, Q_{n c h, h}\right)
\end{aligned}
$$

where $P_{n c h, \max }\left(Q_{n c h, h}, Q_{n c h, c}\right)$ and $P_{n c h, \min }\left(Q_{n c h, h}, Q_{n c h, c}\right)$ are respectively the upper and lower limits of the electric power output of the nch-th CCHP; $Q_{n c h, h, \max }\left(P_{n c h}, Q_{n c h, c}\right)$ and $Q_{n c h, h, \min }\left(P_{n c h}, Q_{n c h, c}\right)$ are respectively the upper and lower limits of the heating power output of the nch-th CCHP; $Q_{n c h, c, \max }\left(P_{n c h}, Q_{n c h, h}\right)$ and $Q_{n c h, c, \min }\left(P_{n c h}, Q_{n c h, h}\right)$ are respectively the upper and lower limits of the cooling power output of the $n c h$-th CCHP.

(8) Charge/discharge constraints of the battery storage facility

$$
\begin{gathered}
P_{s, \text { min }} \leq\left|P_{s, i}\right| \leq P_{s, \text { max }} \\
E S_{e, \text { min }} \leq E S_{e, i} \leq E S_{e, \text { max }}
\end{gathered}
$$

where $P_{s, \max }$ and $P_{s, \min }$ are respectively the upper and lower limits of the charging/discharging power of battery storage facilities; $E S_{e, i}$ is the energy state of the battery storage facility in the $i$-th time period; and $E S_{e, \max }$ and $E S_{e, \min }$ are respectively the upper and lower limits of the energy storage space of BESS facilities.

(9) Absorption/release constraints of the heat energy storage facility

$$
\begin{aligned}
& Q_{h s, \text { min }} \leq\left|Q_{h s, i}\right| \leq Q_{h s, \text { max }} \\
& E S_{h, \text { min }} \leq E S_{h, i} \leq E S_{h, \text { max }}
\end{aligned}
$$

where $Q_{h s, \max }$ and $Q_{h s, \min }$ are respectively the upper and lower limits of the heat absorption/release power of the heat energy storage facilities; $E S_{h, i}$ is the energy state of the heat storage facility in the $i$-th period; and $E S_{h, \max }$ and $E S_{h \text {,min }}$ are respectively the upper and lower limits of the energy storage space of the heat energy storage facility.

(10) Exchange power constraint of the tie line

$$
0 \leq\left|P_{x, i}\right| \leq P_{x, \max }
$$

where $P_{x, \max }$ is the limit value of the exchange power for the tie line. Due to the large loss of longdistance transmission of cold and hot energy across regions, only the case of interconnection with the external power supply network through the power tie line is considered here.

\subsection{Configuring Constraints}

In addition to the operational constraints, it is also necessary to meet constraints such as the installation capacity, energy utilization rate, and energy self-balance rate in the process of RIES configuration decision analysis based on production simulation.

(1) Installation capacity constraint, that is, $N<N_{\max }$, where $N$ is the actual installation quantity and $N_{\max }$ is the maximum installation quantity restricted by various conditions, such as the actual site.

(2) Restrictions on the proportion of renewable energy installed capacity. The distributed generation in the RIES should be based on wind power, photovoltaic power, and other renewable energy sources, and the proportion of their power generation in the total power load of the system should not be less than a certain threshold.

(3) Constraints on fossil energy utilization. The RIES needs to prioritize the consumption of clean energy; the use of fossil energy should reflect the characteristics of "high efficiency", and the integrated energy utilization rate should generally meet the prescribed requirements, that is, $\eta_{F} \geq \eta_{0}$. Here, $\eta_{F}$ is the fossil energy utilization rate, and $\eta_{0}$ is the fossil energy utilization threshold.

(4) Constraints of energy self-balance in the region. Considering comprehensively the energy self-balancing requirements of the RIES and the needs of the development and utilization of distributed 
renewable energy in China, this paper advocates that the exchange of electricity quantity between the RIES and the external power grid can be appropriately set within a settlement period, but generally, it should not exceed $20 \%$ of the total electricity quantity, namely:

$$
\frac{\left|E_{e x}\right|}{\left|E_{L}\right|} \leq \eta_{e x}
$$

where $E_{e x}$ is the exchanged electricity; $E_{L}$ is the total power demand of the load; and $\eta_{e x}$ is the threshold of the self-balancing rate.

\subsection{Configuration Decision Process}

For long-term production simulation, multiple typical random scenarios can be generated through scene clustering, and each typical random scenario is analyzed separately. Then, the distribution probability of each typical scenario is combined to obtain the expected value of the final configuration scheme. Furthermore, since the rated capacity and the number of distributed generations are integers, the range of decision variables in the configuration process should be in the integer range, so the above optimization problem is essentially a mixed integer programming problem. This paper uses a genetic algorithm to solve the optimal configuration scheme in each typical scenario. The specific process is shown in Figure 2, where $N_{m}$ is the amount of typical scenarios.

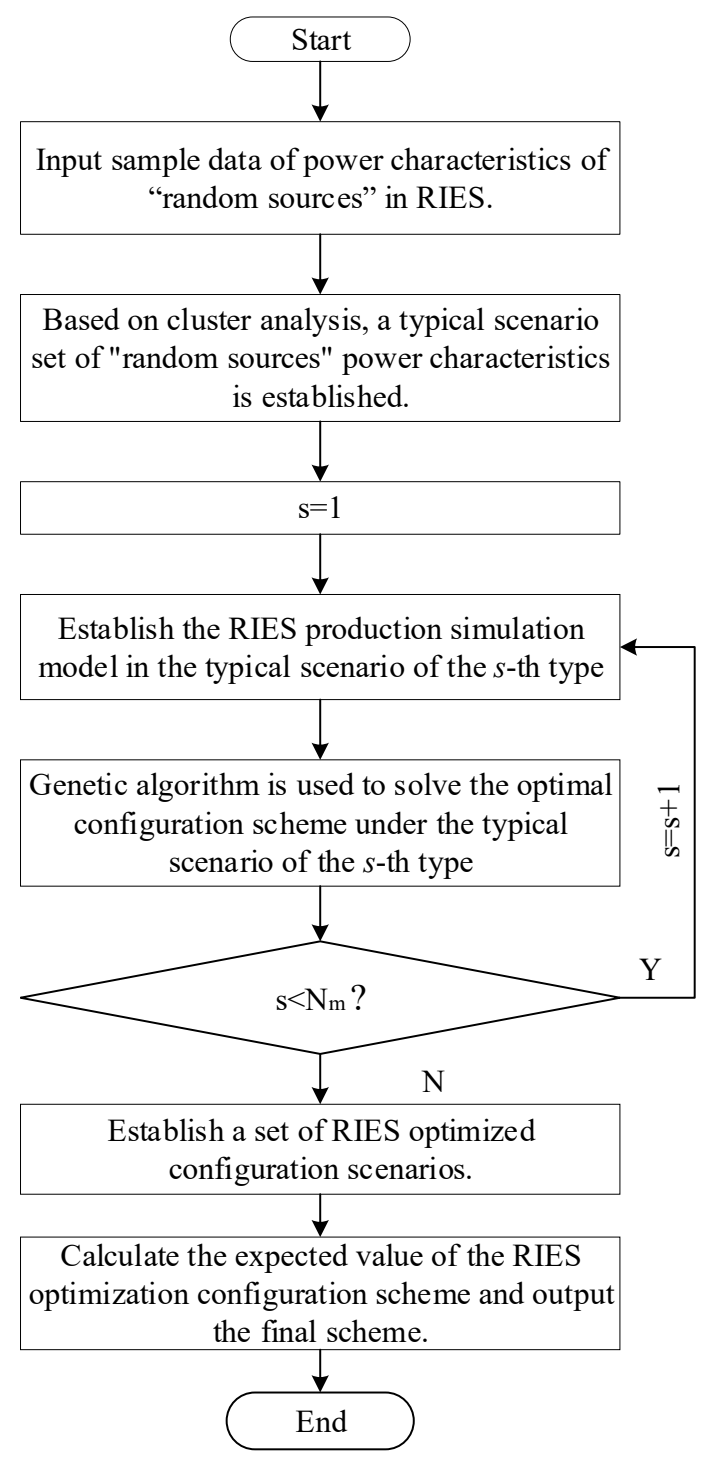

Figure 2. Decision process of optimal configuration. 


\section{Example Analysis}

The typical scenarios of RIES configuration in an industrial park of China to be built are shown in Figures 3-6 through the analysis of historical sample data of resources and load in this region. The corresponding distribution probabilities of each typical scenario are $0.2,0.15,0.35$, and 0.3 . The benchmark capacity of the WG and PV scenarios is $10 \mathrm{MW}$.

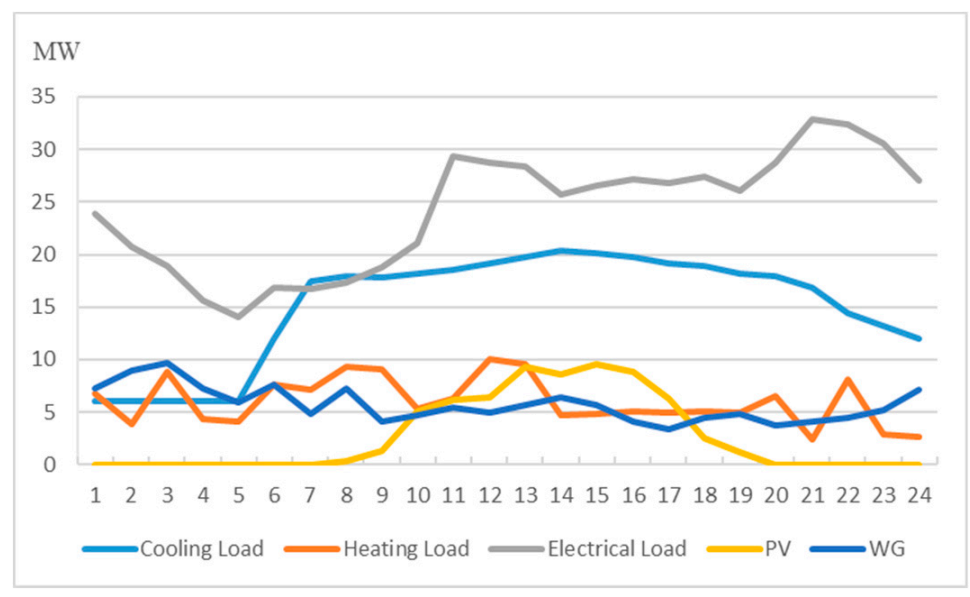

Figure 3. Typical scenario 1 (MW).

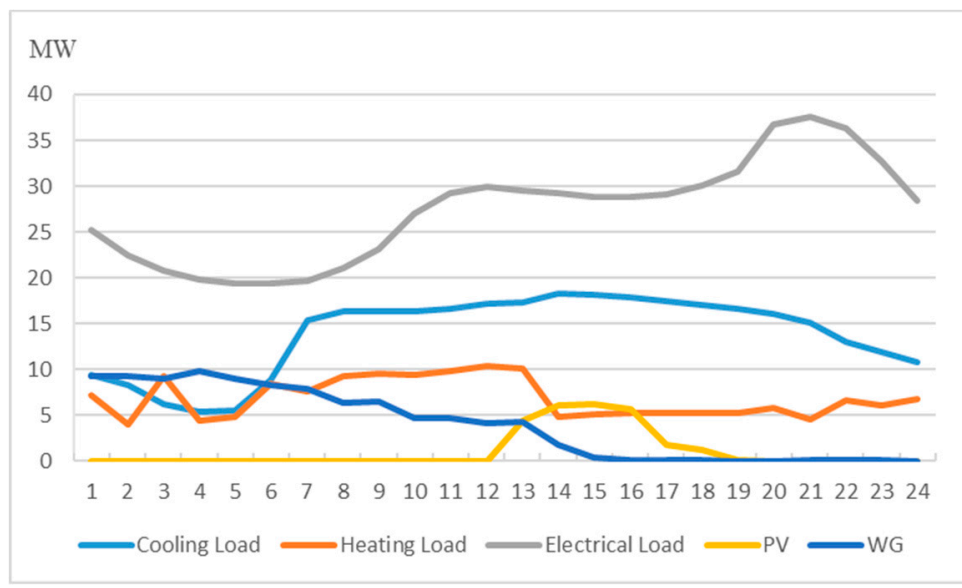

Figure 4. Typical scenario $2(\mathrm{MW})$.

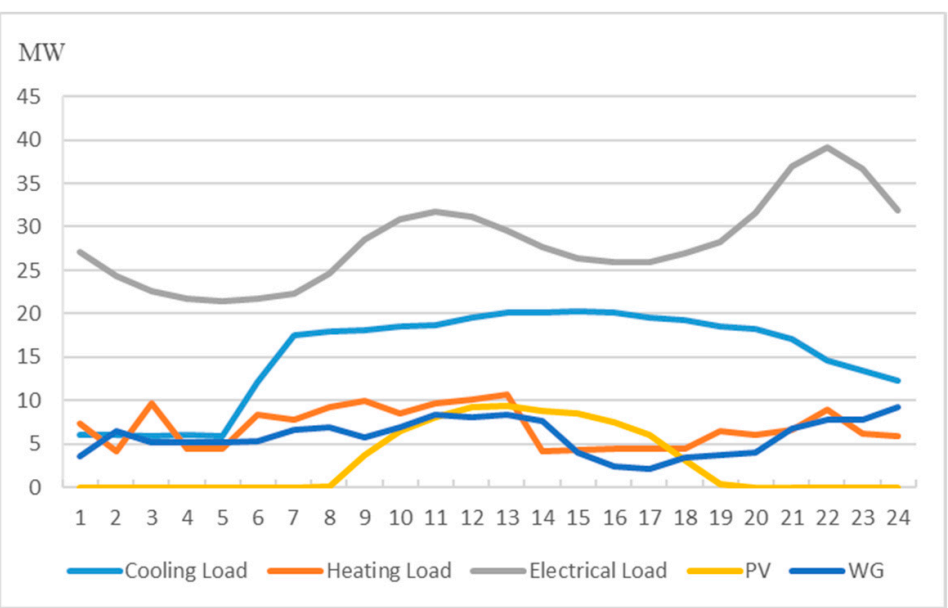

Figure 5. Typical scenario $3(\mathrm{MW})$. 


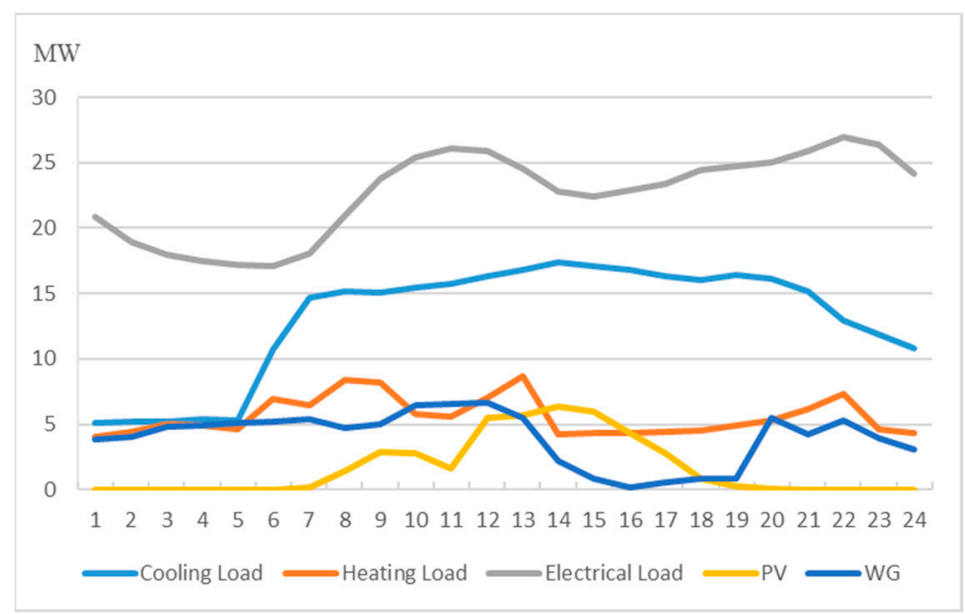

Figure 6. Typical scenario 4 (MW).

The design life of the RIES is considered to be 20 years. The technical and economic parameters of the distributed energy are provided by the construction bid and equipment supply, which are shown as follows

(1) PV: power generation unit capacity $500 \mathrm{~kW}$, cost 2.25 million RMB;

(2) WG: single wind turbine capacity $1 \mathrm{MW}$, cost 9.2 million RMB;

(3) BESS: battery unit capacity $500 \mathrm{~kW} / \mathrm{kWh}$, cost 1.44 million RMB;

(4) Ground source heat pump (GSHP): single unit capacity $600 \mathrm{~kW}$, cost 2 million RMB;

(5) CHP based on micro gas turbine: power generation efficiency $27.1 \%$, thermoelectric ratio 0.64 , single unit capacity $500 \mathrm{~kW}$, cost 1.82 million RMB;

(6) Absorption chiller: single unit cooling capacity $1160 \mathrm{~kW}$, cooling coefficient $K_{c}=2.5$, cost 0.8 million RMB;

(7) Electric boiler: single unit capacity $5 \mathrm{MW}$, cost 1 million RMB;

(8) Air conditioning equipment: single unit capacity $3550 \mathrm{~W}$, cost $3000 \mathrm{RMB}$;

(9) Heat storage tank: per unit capacity cost 0.5 million RMB/MWh.

According to constraints such as the geographical environment, energy utilization ratio, and energy self-balance, the RIES configuration scheme should meet the following requirements:

(1) The installed capacity of PV shall be no more than $30 \mathrm{MW}$;

(2) The installed capacity of WG shall be no more than $20 \mathrm{MW}$;

(3) Comprehensive utilization rate of fossil energy $\eta_{F} \geq 70 \%$;

(4) The proportion of electricity generated by clean energy shall not be less than $60 \%$;

(5) Energy self-balancing rate $1-\frac{\left|E_{e x}\right|}{\left|E_{L}\right|} \geq 80 \%$.

Based on the above model parameters and typical daily scenarios, the RIES configuration scheme under each planning scenario can be obtained through production simulation calculations combined with genetic algorithms, as shown in Table 1.

From Table 1, we can see that the four typical scenarios correspond to the optimal configuration plan of the RIES and calculate the expected value of each distributed energy capacity configuration based on the random probability distribution of each typical scenario. Then, the final RIES configuration plan can be obtained. On this basis, the investment cost of each distributed energy is calculated as follows: 135 million RMB of PV, 184 million RMB of WG, 2.5 million RMB of heat storage tank, 14.4 million RMB of BESS, 33.1 million RMB of CHP, 13 million RMB of absorption chiller, 13.9 million RMB of GSHP, and total investment of distributed energy of 395.94 million RMB. If the traditional deterministic configuration method is used for this configuration problem, scenario 3 will be usually 
selected as the maximum demand scenario to meet the requirements of system adequacy. In this way, the total investment of distributed energy will be 401.87 million RMB, which is 5.93 million more than the expected investment. Thus, it can be seen that the optimal configuration method considering the randomness of scenarios can reduce the total investment cost while meeting the expectation of energy balance of the RIES.

Table 1. Configuration schemes and their expected values under each typical scenario (MW). BESS: battery energy storage system, CHP: combined heat and power, GSHP: ground source heat pump, PV: photovoltaic power generation, WG: wind power generation.

\begin{tabular}{cccccc}
\hline Type & Scenario 1 & Scenario 2 & Scenario 3 & Scenario 4 & Expected Value \\
\hline PV & 30.00 & 30.00 & 30.00 & 30.00 & 30.00 \\
WG & 20.00 & 20.00 & 20.00 & 20.00 & 20.00 \\
Electrical Boiler & 0.00 & 0.00 & 0.00 & 0.00 & 0.00 \\
Heat Storage Tank & 5.00 & 5.00 & 5.00 & 5.00 & 5.00 \\
BESS & 5.00 & 5.00 & 5.00 & 5.00 & 5.00 \\
CHP & 8.50 & 10.00 & 10.00 & 8.00 & 9.10 \\
Absorption Chiller & 19.72 & 18.56 & 19.72 & 17.4 & 18.85 \\
GSHP & 4.80 & 1.80 & 4.80 & 4.20 & 4.17 \\
\hline
\end{tabular}

\section{Conclusions}

Aiming at the problem of optimal configuration of a RIES, this paper proposes a production simulation method for the cluster analysis of random scenarios. First, this method quantifies the time series and random characteristics of RIES configuration scenarios through time series clustering analysis and establishes a set of typical RIES configuration scenarios. Second, considering the constraints of configuration and operation, the production simulation model of an RIES is established with the goal of economy and environmental protection. Finally, based on the set of RIES configuration scenarios, the expected value of the RIES configuration scheme is obtained through production simulation. The accuracy and effectiveness of the above methods are verified by the analysis of practical engineering cases. The research shows that compared to the traditional method, the optimal configuration method allows a finer sizing of the different elements, therefore reducing the risks of high costs due to over-sizing the RIES system. At the same time, the limitation of this method is that it needs a lot of statistical data analysis to ensure the representativeness of typical scenarios. Therefore, a certain statistical risk exists. In addition, the simulations are only based on a $24-\mathrm{h}$ period by space limitation. However, in the actual planning decision-making, a longer time scale is usually chosen. Therefore, in the future, the influence of multiple random factors on the production process of RIES will be further studied. On this basis, a random production simulation method for RIES that is suitable for long-term process planning research will be proposed.

Author Contributions: This research was a collaborative effort between the authors. T.S. proposed the method framework of the integrated energy system configuration and wrote the paper. R.-M.H. provided an optimization strategy for the production simulation of integrated energy system. C.-B.D. performed a case study and reviewed the paper. All authors have read and agreed to the published version of the manuscript.

Funding: This research was funded as a project of the State Grid Shanghai Municipal Electric Power Company (grant number SGTYHT/18-JS-209), named "Optimal configuration and application analysis of urban integrated energy system".

Conflicts of Interest: The authors declare no conflict of interest.

\section{References}

1. Zhou, X.X. New generation energy system under energy revolution. State Grid 2015, 8, 58-61.

2. Jia, H.J.; Mu, Y.F.; Yu, X.D. Thought about the integrated energy system in China. Electr. Power Constr. 2015, $48,16-26$. 
3. Wang, W.L.; Wang, D.; Jia, H.J.; Chen, Z.; Guo, B.; Zhou, H.; Fan, M. Review of steady-state analysis of typical regional integrated energy system in the context of energy Internet. Chin. J. Electr. Eng. 2016, 36, 3292-3304.

4. Rong, A.Y.; Lajdelma, R. An efficient linear programming model and algorithm for trigeneration. Appl. Energy 2005, 82, 40-63. [CrossRef]

5. Mehleri, E.D.; Sarimveis, H.; Markatos, N.C.; Papageorgiou, L.G. A mathematical programming approach for optimal design of distributed energy system at the neighbourhood level. Energy 2012, 44, 96-104. [CrossRef]

6. Wang, J.J.; Jing, Y.Y.; Zhang, C.F. Optimization of capacity and operation for CCHP system by genetic algorithm. Appl. Energy 2010, 87, 1325-1335. [CrossRef]

7. Wang, J.J.; Zhai, Z.Q.; Jing, Y.Y.; Zhang, C. Optimization design of BCHP system to maximize to save energy and reduce environmental impact. Energy 2010, 35, 3388-3398. [CrossRef]

8. Meybodi, M.A.; Behnia, M. Impact of carbon tax on internal combustion engine size selection in a medium scale CHP system. Appl. Energy 2011, 88, 5153-5163. [CrossRef]

9. Carvalho, M.; Serra, L.M.; Lozano, M.A. Optimal synthesis of trigeneration system subject to environmental constraints. Energy 2011, 36, 3779-3790. [CrossRef]

10. Wang, J.J.; Jing, Y.Y.; Zhang, C.F.; Shi, G.H.; Zhang, X.T. A fuzzy multi-criteria decision-making model for trigeneration system. Energy Policy 2008, 36, 3823-3832. [CrossRef]

11. Zhou, Z.; Liu, P.; Li, Z.; Ni, W. An engineering approach to the optimal design of distributed energy system in China. Appl. Therm. Eng. 2012, 53, 387-396. [CrossRef]

12. Li, J.H.; Sang, C.C. Discussion on optimal planning and operation framework for integrated energy system. Electr. Power Constr. 2015, 36, 41-48.

13. Zheng, L.K.; Wang, X.L.; Jiang, B.C. Multi-Objective Optimal Configuration of the CCHP System. Processes 2020, 8, 351. [CrossRef]

14. Sun, L.X.; JU, P.; BAI, J.T.; Tiantian, L.I. Multi-objective economic optimal operation of microgrid based on combined cooling, heating and power considering battery life. Power Gener. Technol. 2020, 41, 64-72.

(C) 2020 by the authors. Licensee MDPI, Basel, Switzerland. This article is an open access article distributed under the terms and conditions of the Creative Commons Attribution (CC BY) license (http://creativecommons.org/licenses/by/4.0/). 